ISAHP 1999, Kobe, Japan, August 12-14, 1999

\title{
THE THEORY AND ALGORITHMS OF CYCLE SYSTEMS IN ANP
}

\author{
Wang Lianfen \\ Dept. of Information, Renmin University of China Sun Hongcai \\ Sun Hongcai \\ Beijing Institute of Decision Science and Management \\ N-24 Tai pin Road, Beijing 100850, P.R.China \\ E-mail: hcs@cenpok.net
}

\begin{abstract}
In this paper, we will discuss the theory and algorithms for cycle systems, including the inner dependent and inner independent cycle systems in ANP. The structure and eigenvalues of system's supermatrioc will be analysed and the algorithm named, power method with original shift, an efficient method for solving the maximum eigenvalue, will be used to get the final periority weights of elements, in system
\end{abstract}

Keywords cycle systems power method with original shift 